\title{
Inexact Inertial Proximal Algorithm for Maximal Monotone Operators
}

\author{
Hadi Khatibzadeh and Sajad Ranjbar
}

\begin{abstract}
In this paper, convergence of the sequence generated by the inexact form of the inertial proximal algorithm is studied. This algorithm which is obtained by the discretization of a nonlinear oscillator with damping dynamical system, has been introduced by Alvarez and Attouch (2001) and Jules and Maingé (2002) for the approximation of a zero of a maximal monotone operator. We establish weak and strong convergence results for the inexact inertial proximal algorithm with and without the summability assumption on errors, under different conditions on parameters. Our theorems extend the results on the inertial proximal algorithm established by Alvarez and Attouch (2001) and Jules and Maingé (2002) as well as the results on the standard proximal point algorithm established by Brézis and Lions (1978), Lions (1978), Djafari Rouhani and Khatibzadeh (2008) and Khatibzadeh (2012). We also answer questions of Alvarez and Attouch (2001).
\end{abstract}

\section{Introduction and preliminaries}

Let $H$ be a real Hilbert space with inner product (.,.) and norm |.|. We denote weak convergence in $H$ by $\rightarrow$ and strong convergence by $\rightarrow$. Let $A$ be a nonempty subset of $H \times H$ to which we shall refer as a (nonlinear) possibly multivalued operator in $H$. $A$ is called monotone (resp. strongly

Key Words: Inertial proximal algorithm, Maximal monotone, Ergodic theorem, Weak convergence, Strong convergence, Subdifferential.

2010 Mathematics Subject Classification: Primary 65Q10; Secondary 65K10, 47H05.

Received: January, 2014.

Revised: May, 2014.

Accepted: May, 2014. 
monotone) iff $\left(y_{2}-y_{1}, x_{2}-x_{1}\right) \geq 0$ (resp. $\left(y_{2}-y_{1}, x_{2}-x_{1}\right) \geq \alpha\left|x_{2}-x_{1}\right|^{2}$ for some $\alpha>0$ ), for all $\left[x_{i}, y_{i}\right] \in A, i=1,2$. $A$ is maximal monotone if $A$ is monotone and $R(I+A)=H$, where $I$ is the identity operator on $H$. Given any function $\varphi: H \rightarrow]-\infty,+\infty$ ] (not necessarily convex) with the domain $D(\varphi)$, its subdifferential is the multivalued operator $\partial \varphi$, defined as

$$
\partial \varphi(x):=\{w \in H \mid \varphi(x)-\varphi(y) \leq(w, x-y), \quad \forall y \in H\} .
$$

The function $\varphi$ is called proper iff there exists $x \in H$ such that $\varphi(x)<+\infty$. It is a well-known result that if $\varphi$ is a proper, convex, and lower semicontinuous function, then $\partial \varphi$ is a maximal monotone operator. We refer the reader to the book by Morosanu [15] in order to understand monotone operators and subdifferential of convex functions in Hilbert spaces.

The most popular algorithm for approximation of a zero of a maximal monotone operator is the following algorithm

$$
\left\{\begin{array}{l}
u_{n+1}=J_{\lambda_{n+1}}\left(u_{n}+e_{n}\right), \quad n \geq 0 \\
u_{0} \in H,
\end{array}\right.
$$

where $J_{\lambda}=(I+\lambda A)^{-1}, A$ is a maximal monotone operator, $\left\{\lambda_{n}\right\}$ is a positive real sequence and $\left\{e_{n}\right\}$ is a suitable sequence in $H$. This iterative scheme is called the proximal point algorithm and was introduced by Martinet in [14] with $\lambda_{n} \equiv \lambda$ and then generalized by Rockafellar [16]. The iterative method (1.1) is also a discrete version of the following first order nonhomogeneous evolution equation of maximal monotone type

$$
\left\{\begin{array}{l}
u^{\prime}(t)+A u(t) \ni f(t) \\
u(0)=u_{0} \in \overline{D(A)} .
\end{array}\right.
$$

In $[1-5,9]$ the authors considered the following iterative method for the approximation of a zero of a maximal monotone operator $A$.

$$
\left\{\begin{array}{l}
u_{n+1}=J_{\lambda_{n}}\left(u_{n}+\alpha_{n}\left(u_{n}-u_{n-1}\right)\right) \\
u_{0}, u_{1} \in H
\end{array}\right.
$$

where $\alpha_{n}$ (resp. $\lambda_{n}$ ) is nonnegative (resp. positive) sequence. They obtained some results on the convergence of $u_{n}$ to a zero of the operator $A$ under suitable assumptions on $\lambda_{n}$ and $\alpha_{n}$. Obviously, the algorithm (1.1) is a special case of (1.3) when $\alpha_{n} \equiv 0$. Jules and Maingé in [9] showed that the iterative method (1.3) provide a better rate of convergence to a zero of $A$, than the standard proximal point algorithm. The iterative method (1.3) is called inertial 
proximal algorithm and can be considered as a discrete version of nonlinear oscillator with damping dynamical system

$$
\left\{\begin{array}{l}
u^{\prime \prime}(t)+\gamma u^{\prime}(t)+A u(t) \ni 0 \\
u(0)=u_{0}, \quad u^{\prime}(0)=u_{1}
\end{array}\right.
$$

where $A$ is a general maximal monotone operator and $\gamma$ is a real positive number. In [2] Alvarez and Attouch proposed two open questions. The first one was related to the convergence of the inexact version of (1.3) and the other one was to develop a general theory to guide the choice of the parameters $\lambda_{k}$ and $\alpha_{k}$.

In this paper, we consider the inexact version of the inertial proximal algorithm

$$
\left\{\begin{array}{l}
u_{n+1}=J_{\lambda_{n+1}}\left(u_{n}+\alpha_{n}\left(u_{n}-u_{n-1}\right)+e_{n}\right), \\
u_{0}, u_{1} \in H
\end{array}\right.
$$

where $\alpha_{n}$ (resp. $\lambda_{n}$ ) is nonnegative (resp. positive) sequence, $\left\{e_{n}\right\}$ is an error sequence in $H$ and $A$ is a maximal monotone operator. Weak and strong convergence results are proved, under different conditions on parameters and errors. Our theorems extend the results of $[2,6,7,9,11,12]$.

In Section 2 of this paper, we investigate the relation between the boundedness of the sequence $\left\{u_{n}\right\}$ generated by (1.5) and the assumption $A^{-1}(0) \neq \emptyset$. Also the weak convergence of the weighted average of the sequence $\left\{u_{n}\right\}$ is proved. In Section 3, we assume that $\left\{u_{n}\right\}$ is bounded and show that the set of all weak cluster points of the sequence $\left\{u_{n}\right\}$ is a subset of $A^{-1}(0)$. Then we prove the weak convergence theorem for the sequence $\left\{u_{n}\right\}$ that is one of the main results of the paper and extends the weak convergence theorem of Attouch and Alvarez [2]. Section 4 is devoted to the special but important case $A=\partial \varphi$, where $\varphi$ is a proper, convex and lower semicontinuous function. We prove the weak convergence of the sequence $\left\{u_{n}\right\}$ generated by (1.5) when $A=\partial \varphi$ with different conditions on parameters and error. In Section 5 , the strong convergence of the sequence is studied when the maximal monotone operator $A$ is strongly monotone or the resolvent of $A$ is a compact operator.

We recall some notations, definitions and lemmas that we need in the sequel.

Definition 1.1. Given a bounded sequence $\left\{u_{n}\right\}$ in $H$, the asymptotic center $c$ of $\left\{u_{n}\right\}$ is defined as follows (see [8]): for every $q \in H$, let $\varphi(q)=$ $\lim _{n \rightarrow \infty} \sup \left|u_{n}-q\right|^{2}$. Then $\varphi$ is a continuous and strictly convex function on $H$, satisfying $\varphi(q) \rightarrow \infty$ as $|q| \rightarrow \infty$. Thus $\varphi$ achieves its minimum on $H$ at a unique point $c$, called the asymptotic center of the sequence $\left\{u_{n}\right\}$. 
Lemma 1.2. [11] Suppose that $\left\{\alpha_{n}\right\}$ is a nonnegative sequence and $\left\{\lambda_{n}\right\}$ is a positive sequence such that $\sum_{n=1}^{+\infty} \lambda_{n}=+\infty$. If $\frac{\alpha_{n}}{\lambda_{n}} \rightarrow 0$ as $n \rightarrow+\infty$, then $\frac{\sum_{k=1}^{n} \alpha_{k}}{\sum_{k=1}^{n} \lambda_{k}} \rightarrow 0$ as $n \rightarrow+\infty$.

Lemma 1.3. [13] Let $\left\{\phi_{n}\right\} \subset\left[0,+\infty\left[\right.\right.$ and $\left\{\delta_{n}\right\} \subset[0,+\infty[$ satisfy

(a) $\phi_{n+1}-\phi_{n} \leq \theta_{n}\left(\phi_{n}-\phi_{n-1}\right)+\delta_{n}$,

(b) $\sum_{n} \delta_{n}<+\infty$

(c) $\left\{\theta_{n}\right\} \subset[0, \theta[$, where $\theta \in[0,1[$.

Then $\left\{\phi_{n}\right\}$ is a converging sequence and $\sum_{n}\left[\phi_{n+1}-\phi_{n}\right]_{+}<\infty$, where $[t]_{+}:=$ $\max \{t, 0\} \forall t \in \mathbb{R}$.

Lemma 1.4. Suppose that $a_{n}$ and $b_{n}$ are nonnegative real sequences and that $\sum_{n=1}^{+\infty} b_{n}<+\infty$. If $a_{n+1} \leq a_{n}+b_{n}$, for all $n \geq 1$, then there exists $\lim _{n} a_{n}$.

Notation 1.5. Throughout the paper $\varphi$ is a proper, convex and lower semicontinuous function, $A$ is a maximal monotone operator and $w_{n}:=\left(\sum_{i=1}^{n} \lambda_{i+1}\right)^{-1} \sum_{i=1}^{n} \lambda_{i+1} u_{i+1}$ is the weighted average of the sequence $\left\{u_{n}\right\}$. We denote by $\omega_{w}\left(x_{n}\right)$ the set of all weak cluster points of the sequence $x_{n}$, and by $A u_{n+1}$ the element $\frac{u_{n}-u_{n+1}+\alpha_{n}\left(u_{n}-u_{n-1}\right)+e_{n}}{\lambda_{n+1}}$ in $H$.

\section{Boundedness of the Sequence and an Ergodic Theo- rem}

In this section we first study the relation between boundedness of the sequence $\left\{u_{n}\right\}$ and the assumption $A^{-1}(0) \neq \emptyset$ as well as the existence of $\lim \left|u_{n}-p\right|$ for each $p \in A^{-1}(0)$. Then an ergodic theorem for the weak convergence of the weighted average of the sequence $\left\{u_{n}\right\}$ is studied.

Proposition 2.1. Suppose $A^{-1}(0) \neq \emptyset$ and $\left\{u_{n}\right\}$ is given by (1.5).

(a) If $e_{n} \equiv 0$ and

$$
\left\{\begin{array}{l}
\left(\alpha_{1}\right) \exists \alpha \in\left[0,1\left[\quad \text { such that } \quad \forall k \in \mathbb{N}, 0 \leq \alpha_{k} \leq \alpha\right.\right. \\
\left(\alpha_{2}\right) \sum_{n=1}^{+\infty} \alpha_{n}\left|u_{n}-u_{n-1}\right|^{2}<+\infty
\end{array}\right.
$$

then $\lim _{n}\left|u_{n}-p\right|$ exists and $\sum_{n=1}^{\infty}\left[\left|u_{n}-p\right|-\left|u_{n-1}-p\right|\right]_{+}<\infty, \forall p \in A^{-1}(0)$. (b) If $\left\{u_{n}\right\}$ is bounded, $\left(E_{1}\right) \sum_{n=1}^{+\infty}\left|e_{n}\right|<+\infty$ and $\left(\alpha_{1}\right)$ and $\left(\alpha_{2}\right)$ are satisfied, then for all $p \in A^{-1}(0), \lim _{n}\left|u_{n}-p\right|$ exists and $\sum_{n=1}^{\infty}\left[\left|u_{n}-p\right|-\left|u_{n-1}-p\right|\right]_{+}<$ $\infty$.

(c) If $\left(E_{1}\right) \sum_{n=1}^{+\infty}\left|e_{n}\right|<+\infty$ and $\sum_{n=1}^{+\infty} \alpha_{n}\left|u_{n}-u_{n-1}\right|<+\infty$, then for all $p \in A^{-1}(0), \lim _{n}\left|u_{n}-p\right|$ exists. 
Proof. In order to prove (a), see [2, Theorem 2.1]. Let us prove (b). Suppose that $p \in A^{-1}(0)$, then by (1.5) and the monotonicity of $A$

$0 \geq\left(u_{n+1}-u_{n}-\alpha_{n}\left(u_{n}-u_{n-1}\right)-e_{n}, u_{n+1}-p\right)$

$=\left(u_{n+1}-u_{n}, u_{n+1}-p\right)-\alpha_{n}\left(u_{n}-u_{n-1}, u_{n+1}-u_{n}\right)-\alpha_{n}\left(u_{n}-u_{n-1}, u_{n}-p\right)-$

$\left(e_{n}, u_{n+1}-p\right)$

$=\frac{1}{2}\left|u_{n+1}-p\right|^{2}-\frac{1}{2}\left|u_{n}-p\right|^{2}-\frac{\alpha_{n}^{2}}{2}\left|u_{n}-u_{n-1}\right|^{2}+\frac{1}{2}\left|u_{n+1}-u_{n}-\alpha_{n}\left(u_{n}-u_{n-1}\right)\right|^{2}-$ $\frac{\alpha_{n}}{2}\left|u_{n}-u_{n-1}\right|^{2}-\frac{\alpha_{n}}{2}\left|u_{n}-p\right|^{2}+\frac{\alpha_{n}}{2}\left|u_{n-1}-p\right|^{2}-\left(e_{n}, u_{n+1}-p\right)$.

Set $\phi_{n}=\frac{1}{2}\left|u_{n}-p\right|^{2}$. We have

$\phi_{n+1}-\phi_{n}-\alpha_{n}\left(\phi_{n}-\phi_{n-1}\right) \leq \alpha_{n}\left|u_{n}-u_{n-1}\right|^{2}+\left|e_{n}\right|\left|u_{n+1}-p\right|$.

Hence by Lemma 1.3, $\left(\alpha_{2}\right)$ and $\left(E_{1}\right)$, there exists $\lim _{n}\left|u_{n}-p\right|$ and

$\sum_{n=1}^{\infty}\left[\left|u_{n}-p\right|-\left|u_{n-1}-p\right|\right]_{+}<\infty$.

Now we prove (c). By nonexpansivity of the resolvent operator and (1.5), we have

$\left|u_{n+1}-p\right| \leq\left|u_{n}-p\right|+\alpha_{n}\left|u_{n}-u_{n-1}\right|+e_{n}$,

thus by Lemma 1.4, $\left(\alpha_{3}\right)$ and $\left(E_{1}\right), \lim _{n}\left|u_{n}-p\right|$ exists.

In the following proposition, under the suitable conditions, we prove that the boundedness of the sequence $\left\{u_{n}\right\}$ generated by $(1.5)$ implies $A^{-1}(0) \neq \emptyset$. Also, we show that the set of weak cluster points of the weighted average of the sequence $u_{n}$, say $w_{n}$, is a subset of $A^{-1}(0)$.

Proposition 2.2. Let $\left\{u_{n}\right\}$ be a bounded sequence generated by (1.5). If the following conditions hold:

$$
\left\{\begin{array}{l}
\left(\Lambda_{1}\right) \sum_{n=1}^{+\infty} \lambda_{n}=+\infty, \\
\left(\alpha_{3}\right) \sum_{n=1}^{+\infty} \alpha_{n}\left|u_{n}-u_{n-1}\right|<+\infty \text { or }\left(\alpha_{4}\right) \frac{\alpha_{n}\left|u_{n}-u_{n-1}\right|}{\lambda_{n+1}} \rightarrow 0, \\
\left(E_{1}\right) \sum_{n=1}^{+\infty}\left|e_{n}\right|<+\infty \text { or }\left(E_{2}\right) \frac{\left|e_{n}\right|}{\lambda_{n+1}} \rightarrow 0,
\end{array}\right.
$$

then $A^{-1}(0) \neq \emptyset$ and $\omega_{w}\left(w_{n}\right) \subset A^{-1}(0)$, where $\omega_{w}\left(w_{n}\right)$ is the set of weak cluster points of $w_{n}$.

Proof. Suppose $[x, y] \in A$, since $\left\{u_{n}\right\}$ is bounded, there is a subsequence $\left\{w_{n_{j}}\right\}$ of $\left\{w_{n}\right\}$ such that $w_{n_{j}} \rightarrow p \in H$. On the other hand, by monotonicity of $A$, we get

$$
\begin{aligned}
& \left(x-w_{n_{j}}, y\right)=\left(x-\left(\sum_{i=0}^{n_{j}-1} \lambda_{i+1}\right)^{-1} \sum_{i=0}^{n_{j}-1} \lambda_{i+1} u_{i+1}, y\right) \\
& =\left(\sum_{i=0}^{n_{j}-1} \lambda_{i+1}\right)^{-1} \sum_{i=0}^{n_{j}-1} \lambda_{i+1}\left(x-u_{i+1}, y\right) \\
& =\left(\sum_{i=0}^{n_{j}-1} \lambda_{i+1}\right)^{-1} \sum_{i=0}^{n_{j}-1} \lambda_{i+1}\left(\left(x-u_{i+1}, y-A u_{i+1}\right)+\left(x-u_{i+1}, A u_{i+1}\right)\right) \\
& \geq\left(\sum_{i=0}^{n_{j}-1} \lambda_{i+1}\right)^{-1} \sum_{i=0}^{n_{j}-1}\left(x-u_{i+1}, \lambda_{i+1} A u_{i+1}\right) \\
& =\left(\sum_{i=0}^{n_{j}-1} \lambda_{i+1}\right)^{-1} \sum_{i=0}^{n_{j}-1}\left(x-u_{i+1}, u_{i}-u_{i+1}+\alpha_{i}\left(u_{i}-u_{i-1}\right)+e_{i}\right) \\
& =\left(\sum_{i=0}^{n_{j}-1} \lambda_{i+1}\right)^{-1} \sum_{i=0}^{n_{j}-1}\left(x-u_{i+1},\left(1+\alpha_{i}\right)\left(u_{i}-x\right)-\alpha_{i}\left(u_{i-1}-x\right)+x-u_{i+1}+e_{i}\right) \\
& =\left(\sum_{i=0}^{n_{j}-1} \lambda_{i+1}\right)^{-1} \sum_{i=0}^{n_{j}-1}\left[-\left(1+\alpha_{i}\right)\left(u_{i+1}-x, u_{i}-x\right)+\alpha_{i}\left(u_{i+1}-x, u_{i-1}-\right.\right.
\end{aligned}
$$


$\left.x)+\left|u_{i+1}-x\right|^{2}+\left(x-u_{i+1}, e_{i}\right)\right]$

$=\left(\sum_{i=0}^{n_{j}-1} \lambda_{i+1}\right)^{-1} \sum_{i=0}^{n_{j}-1}\left[-\left(u_{i+1}-x, u_{i}-x\right)+\alpha_{i}\left(u_{i+1}-x, u_{i-1}-u_{i}\right)+\right.$

$\left.\left|u_{i+1}-x\right|^{2}+\left(x-u_{i+1}, e_{i}\right)\right]$

$\geq\left(\sum_{i=0}^{n_{j}-1} \lambda_{i+1}\right)^{-1} \sum_{i=0}^{n_{j}-1}\left[\frac{1}{2}\left|u_{i+1}-x\right|^{2}-\frac{1}{2}\left|u_{i}-x\right|^{2}+\frac{1}{2}\left|u_{i+1}-u_{i}\right|^{2}-\right.$

$\left.\alpha_{i}\left|u_{i+1}-x\right|\left|u_{i}-u_{i-1}\right|-\left|e_{i}\right|\left|u_{i+1}-x\right|\right]$

$\geq\left(\sum_{i=0}^{n_{j}-1} \lambda_{i+1}\right)^{-1} \sum_{i=0}^{n_{j}-1}\left[\frac{1}{2}\left(\left|u_{i+1}-x\right|^{2}-\left|u_{i}-x\right|^{2}\right)-\alpha_{i}\left|u_{i+1}-x\right|\left|u_{i}-u_{i-1}\right|-\left|e_{i}\right|\left|u_{i+1}-x\right|\right]$

$\geq\left(\sum_{i=0}^{n_{j}-1} \lambda_{i+1}\right)^{-1}\left[-\frac{1}{2}\left|u_{0}-x\right|^{2}-\sum_{i=0}^{n_{j}-1} \alpha_{i}\left|u_{i+1}-x\right|\left|u_{i}-u_{i-1}\right|-\sum_{i=0}^{n_{j}-1}\left|e_{i}\right|\left|u_{i+1}-x\right|\right]$.

Letting $j \rightarrow \infty$, by Lemma 1.1 and (2.2), we get $(x-p, y) \geq 0$. Thus by maximality of $A$, we have $p \in A^{-1}(0)$, as desired.

Remark 2.3. If $e_{n} \equiv 0$ in (1.5) and the conditions $\left(\alpha_{1}\right),\left(\alpha_{2}\right)$, [ $\left(\alpha_{3}\right)$ or $\left.\left(\alpha_{4}\right)\right]$ and $\left(\Lambda_{1}\right)$ are satisfied, then by Proposition 2.2 and part (a) of Proposition 2.1, $\left\{u_{n}\right\}$ is bounded if and only if $A^{-1}(0) \neq \emptyset$.

Theorem 2.4. Let $\left\{u_{n}\right\}$ be a bounded sequence generated by (1.5). Suppose that the conditions $\left(\alpha_{1}\right),\left(\alpha_{2}\right),\left[\left(\alpha_{3}\right)\right.$ or $\left.\left(\alpha_{4}\right)\right]$ and $\left(\Lambda_{1}\right)$ are satisfied. If $\left(E_{1}\right) \sum_{n=1}^{+\infty}\left|e_{n}\right|<+\infty$, then $w_{n} \rightarrow p \in A^{-1}(0)$ as $n \rightarrow \infty$, which is also the asymptotic center of $\left\{u_{n}\right\}$.

Proof. By Proposition 2.2, $A^{-1}(0) \neq \emptyset$ and $\omega_{w}\left(w_{n}\right) \subset A^{-1}(0)$, thus by part (b) of Proposition 2.1, there exists $\lim _{n}\left|u_{n}-p\right|, \quad \forall p \in \omega_{w}\left(w_{n}\right)$. We show that $\omega_{w}\left(w_{n}\right)$ is singleton. Suppose $p, q \in \omega_{w}\left(w_{n}\right)$ and $p \neq q$, then there exists $\lim _{n}\left(\left|u_{n}-p\right|^{2}-\left|u_{n}-q\right|^{2}\right)$, hence $\lim _{n \rightarrow+\infty}\left(u_{n}, p-q\right)$ exists. This follows that $\lim _{n \rightarrow+\infty}\left(w_{n}, p-q\right)$ exists. This implies that $(q, p-q)=(p, p-q)$ and hence $p=q$. So, $w_{n} \rightarrow p \in A^{-1}(0)$ as $n \rightarrow+\infty$. Now, we show that $p$ is the asymptotic center of $\left\{u_{n}\right\}$. Suppose that $q \in H$ and $q \neq p$, then

$$
\left|u_{n}-p\right|^{2}=\left|u_{n}-q\right|^{2}+2\left(u_{n}, q-p\right)+|p|^{2}-|q|^{2} .
$$

Multiplying both sides of the above equality by $\lambda_{n}$, summing up from $n=1$ to $n=m$ and dividing by $\sum_{n=1}^{m} \lambda_{n}$. Taking limsup as $m \rightarrow+\infty$, we get

$$
\lim _{n \rightarrow+\infty}\left|u_{n}-p\right|^{2}=\limsup _{m \rightarrow+\infty}\left(\sum_{n=1}^{m} \lambda_{n}\right)^{-1}\left(\sum_{n=1}^{m} \lambda_{n}\left|u_{n}-q\right|^{2}\right)-|q-p|^{2}<\limsup _{n \rightarrow+\infty}\left|u_{n}-q\right|^{2} .
$$

It shows that $p$ is the asymptotic center of the sequence $u_{n}$ as desired.

Remark 2.5. By Remark 2.1, if $e_{n} \equiv 0$, we can replace the boundedness of $u_{n}$ by $A^{-1}(0) \neq \emptyset$ in Theorem 2.4 . 


\section{Weak Convergence of the Algorithm with Errors}

The main result of this section is to prove weak convergence of the sequence $\left\{u_{n}\right\}$ generated by (1.5) to a zero of the maximal monotone operator $A$ provided that the suitable assumptions on the parameters $\lambda_{n}$ and $\alpha_{n}$ and appropriate assumptions on the error sequence $\left\{e_{n}\right\}$.

Lemma 3.1. Suppose that $\left\{u_{n}\right\}$ is the bounded sequence generated by (1.5). If the conditions

$$
\left(\alpha_{5}\right) \sum_{n=1}^{+\infty} \frac{\alpha_{n}^{2}\left|u_{n}-u_{n-1}\right|^{2}}{\lambda_{n+1}^{2}}<+\infty \text { and }\left(E_{3}\right) \sum_{n=1}^{+\infty} \frac{\left|e_{n}\right|^{2}}{\lambda_{n+1}^{2}}<+\infty
$$

are satisfied, then there exists $\lim _{n \rightarrow+\infty}\left|A u_{n}\right|$.

Proof. By monotonicity of $A$, we have

$$
\left(A u_{n+1}-A u_{n}, u_{n+1}-u_{n}\right) \geq 0 .
$$

The equation (1.5) implies that

$$
\left(A u_{n+1}-A u_{n}, \alpha_{n}\left(u_{n}-u_{n-1}\right)+e_{n}-\lambda_{n+1} A u_{n+1}\right) \geq 0 .
$$

Then

$$
\begin{aligned}
& \left|A u_{n+1}\right|^{2} \leq\left(A u_{n}, A u_{n+1}\right)+\left(A u_{n+1}-A u_{n}, \frac{\alpha_{n}}{\lambda_{n+1}}\left(u_{n}-u_{n-1}\right)+\frac{e_{n}}{\lambda_{n+1}}\right) \\
& \leq \frac{1}{2}\left|A u_{n}\right|^{2}+\frac{1}{2}\left|A u_{n+1}\right|^{2}-\frac{1}{2}\left|A u_{n}-A u_{n+1}\right|^{2}+\frac{1}{2}\left|A u_{n}-A u_{n+1}\right|^{2} \\
& +\frac{1}{2}\left|\frac{\alpha_{n}}{\lambda_{n+1}}\left(u_{n}-u_{n-1}\right)+\frac{e_{n}}{\lambda_{n+1}}\right|^{2} \\
& \leq \frac{1}{2}\left|A u_{n}\right|^{2}+\frac{1}{2}\left|A u_{n+1}\right|^{2}+\frac{\alpha_{n}^{2}}{\lambda_{n+1}^{2}}\left|u_{n}-u_{n-1}\right|^{2}+\frac{\left|e_{n}\right|^{2}}{\lambda_{n+1}^{2}} .
\end{aligned}
$$

Therefore

$$
\left|A u_{n+1}\right|^{2} \leq\left|A u_{n}\right|^{2}+2 \frac{\alpha_{n}^{2}}{\lambda_{n+1}^{2}}\left|u_{n}-u_{n-1}\right|^{2}+2 \frac{\left|e_{n}\right|^{2}}{\lambda_{n+1}^{2}} .
$$

Then there exists $\lim _{n \rightarrow+\infty}\left|A u_{n}\right|$, by $\left(\alpha_{5}\right),\left(E_{3}\right)$ and Lemma 1.4.

Proposition 3.2. Let $\left\{u_{n}\right\}$ be a bounded sequence generated by (1.5). If $\left(\alpha_{5}\right)$, $\left(E_{3}\right)$, and the following conditions hold:

$$
\left\{\begin{array}{l}
\left(\Lambda_{2}\right) \sum_{n=1}^{+\infty} \lambda_{n}^{2}=+\infty, \\
\left(\alpha_{3}\right) \sum_{n=1}^{+\infty} \alpha_{n}\left|u_{n}-u_{n-1}\right|<+\infty \text { or }\left(\alpha_{6}\right) \frac{\alpha_{n}\left|u_{n}-u_{n-1}\right|}{\lambda_{n+1}^{2}} \rightarrow 0, \\
\left(E_{1}\right) \sum_{n=1}^{+\infty}\left|e_{n}\right|<+\infty \text { or }\left(E_{4}\right) \frac{\left|e_{n}\right|}{\lambda_{n+1}^{2}} \rightarrow 0,
\end{array}\right.
$$

then $\lim _{n \rightarrow+\infty}\left|A u_{n}\right|=0, A^{-1}(0) \neq \emptyset$ and $\omega_{w}\left(u_{n}\right) \subset A^{-1}(0)$. 
Proof. Set $L=\sup _{n \geq 1}\left|A u_{n}\right|$. By Proposition 2.2, $A^{-1}(0) \neq \emptyset$ (because $\left(\Lambda_{2}\right) \Rightarrow\left(\Lambda_{1}\right),\left(\alpha_{5}\right) \Rightarrow\left(\alpha_{4}\right)$ and $\left.\left(E_{3}\right) \Rightarrow\left(E_{2}\right)\right)$. Assume $p \in A^{-1}(0)$. By monotonicity of $A$

$$
\left(\lambda_{n+1} A u_{n+1}, u_{n+1}-p\right) \geq 0 .
$$

By (1.5), we get

$$
\left(u_{n}-u_{n+1}+\alpha_{n}\left(u_{n}-u_{n-1}\right)+e_{n}, u_{n+1}-p\right) \geq 0,
$$

which implies

$$
\left(\alpha_{n}\left(u_{n}-u_{n-1}\right)+e_{n}, u_{n+1}-p\right) \geq\left(u_{n+1}-u_{n}, u_{n+1}-p\right) .
$$

Thus, by (1.5)

$$
\left(\alpha_{n}\left(u_{n}-u_{n-1}\right)+e_{n}, u_{n+1}-p\right) \geq\left(\alpha_{n}\left(u_{n}-u_{n-1}\right)+e_{n}-\lambda_{n+1} A u_{n+1}, u_{n+1}-p\right),
$$

hence

$2 \alpha_{n}\left|u_{n}-u_{n-1}\right|\left|u_{n+1}-p\right|+2\left|e_{n}\right|\left|u_{n+1}-p\right|+\left|u_{n}-p\right|^{2}-\left|u_{n+1}-p\right|^{2}$

$\geq\left|\alpha_{n}\left(u_{n}-u_{n-1}\right)+e_{n}-\lambda_{n+1} A u_{n+1}\right|^{2}=\lambda_{n+1}^{2}\left|\frac{\alpha_{n}}{\lambda_{n+1}}\left(u_{n}-u_{n-1}\right)+\frac{e_{n}}{\lambda_{n+1}}-A u_{n+1}\right|^{2}$

$=\lambda_{n+1}^{2}\left[\left|\frac{\alpha_{n}}{\lambda_{n+1}}\left(u_{n}-u_{n-1}\right)+\frac{e_{n}}{\lambda_{n+1}}\right|^{2}+\left|A u_{n+1}\right|^{2}-2\left(\frac{\alpha_{n}}{\lambda_{n+1}}\left(u_{n}-u_{n-1}\right)+\frac{e_{n}}{\lambda_{n+1}}, A u_{n+1}\right)\right]$

$\geq \lambda_{n+1}^{2}\left[\left|A u_{n+1}\right|^{2}-2\left|\frac{\alpha_{n}}{\lambda_{n+1}}\left(u_{n}-u_{n-1}\right)+\frac{e_{n}}{\lambda_{n+1}}\right|\left|A u_{n+1}\right|\right]$

$\geq \lambda_{n+1}^{2}\left[\left|A u_{n+1}\right|^{2}-2 \frac{\alpha_{n}}{\lambda_{n+1}}\left|u_{n}-u_{n-1}\right|\left|A u_{n+1}\right|-2 \frac{\left|e_{n}\right|}{\lambda_{n+1}}\left|A u_{n+1}\right|\right]$.

So

$$
\begin{aligned}
\lambda_{n+1}^{2}\left|A u_{n+1}\right|^{2} \leq & 2 L \lambda_{n+1} \alpha_{n}\left|u_{n}-u_{n-1}\right|+2 L \lambda_{n+1}\left|e_{n}\right|+2 \alpha_{n}\left|u_{n}-u_{n-1}\right|\left|u_{n+1}-p\right| \\
& +2\left|e_{n}\right|\left|u_{n+1}-p\right|+\left|u_{n}-p\right|^{2}-\left|u_{n+1}-p\right|^{2} .
\end{aligned}
$$

Summing up both sides of (3.3) from $n=1$ to $n=k$ and then dividing by $\sum_{n=1}^{k} \lambda_{n+1}^{2} .\left|A u_{n}\right| \rightarrow l$ as $n \rightarrow+\infty$ (by Lemma 3.1) implies that $\left(\sum_{n=1}^{k} \lambda_{n+1}^{2}\right)^{-1} \sum_{n=1}^{k} \lambda_{n+1}^{2}\left|A u_{n+1}\right|^{2} \rightarrow l$ as $k \rightarrow+\infty$, then by assumptions and Lemma 1.2, we get $l=0$. Now, if $u_{n_{j}} \rightarrow q$, then by demicloseness of $A$, we get $q \in A^{-1}(0)$, hence $\omega_{w}\left(u_{n}\right) \subset A^{-1}(0)$.

Remark 3.3. Proposition 3.2 shows that if $A^{-1}(0)$ is a singleton (which happens if, for example, $A$ is strictly monotone), then $u_{n} \rightarrow p$, where $p$ is the unique element of $A^{-1}(0)$.

Remark 3.4. Although Proposition 3.2 does not imply the weak convergence of $u_{n}$ to $p \in A^{-1}(0)$ unless $A^{-1}(0)$ are singleton, it improves the errors in the inertial proximal algorithm. It shows that the error sequence $\left\{\left|e_{n}\right|\right\}$ can go to infinity, provided that the suitable assumptions on $\lambda_{n}$ and $\alpha_{n}$ hold. For example, $\left|e_{n}\right|=n, \alpha_{n}=\frac{1}{n^{2}}$ and $\lambda_{n}=(n-1)^{2}$ satisfy the assumptions of Proposition 3.2 for any bounded sequence $\left\{u_{n}\right\}$. 
In the following theorem, we obtain the weak convergence of the sequence $\left\{u_{n}\right\}$ generated by (1.5). Our result extends the result of [2, Theorem 2.1].

Theorem 3.5. Let $\left\{u_{n}\right\}$ be a bounded sequence generated by (1.5). Suppose that the conditions $\left(\Lambda_{2}\right),\left(\alpha_{1}\right),\left(\alpha_{2}\right),\left(\alpha_{5}\right),\left(E_{1}\right)$ and $\left(E_{3}\right)$ are satisfied. Then $u_{n} \rightarrow p \in A^{-1}(0)$ as $n \rightarrow \infty$.

Proof. By Proposition 2.2, $A^{-1}(0) \neq \emptyset$. Assume $p \in A^{-1}(0)$. By monotonicity of $A$, we have

$$
\left(u_{n+1}-u_{n}-\alpha_{n}\left(u_{n}-u_{n-1}\right)-e_{n}, u_{n+1}-p\right) \leq 0 .
$$

Thus

$\lambda_{n+1}^{2}\left|A u_{n+1}\right|^{2}+\left|u_{n+1}-p\right|^{2} \leq\left|u_{n}-p+\alpha_{n}\left(u_{n}-u_{n-1}\right)+e_{n}\right|^{2}$

$\leq\left|u_{n}-p\right|^{2}+\alpha_{n}^{2}\left|u_{n}-u_{n-1}\right|^{2}+\left|e_{n}\right|^{2}+2 \alpha_{n}\left(u_{n}-p, u_{n}-u_{n-1}\right)+$

$2 \alpha_{n}\left(e_{n}, u_{n}-u_{n-1}\right)+2\left(u_{n}-p, e_{n}\right)$

$\leq\left|u_{n}-p\right|^{2}+\alpha_{n}^{2}\left|u_{n}-u_{n-1}\right|^{2}+\left|e_{n}\right|^{2}+\alpha_{n}\left|u_{n}-p\right|^{2}+\alpha_{n}\left|u_{n}-u_{n-1}\right|^{2}-$

$\alpha_{n}\left|u_{n-1}-p\right|^{2}+\alpha_{n}\left|u_{n}-u_{n-1}\right|^{2}+\alpha_{n}\left|e_{n}\right|^{2}+2\left|e_{n}\right|\left|u_{n}-p\right|$.

Therefore

$\lambda_{n+1}^{2}\left|A u_{n+1}\right|^{2} \leq\left|u_{n}-p\right|^{2}-\left|u_{n+1}-p\right|^{2}+3 \alpha_{n}\left|u_{n}-u_{n-1}\right|^{2}+$

$\alpha_{n}\left(\left|u_{n}-p\right|^{2}-\left|u_{n-1}-p\right|^{2}\right)+2\left|e_{n}\right|^{2}+2\left|e_{n}\right|\left|u_{n}-p\right|$

$\leq\left|u_{n}-p\right|^{2}-\left|u_{n+1}-p\right|^{2}+3 \alpha_{n}\left|u_{n}-u_{n-1}\right|^{2}+$

$\alpha\left[\left|u_{n}-p\right|^{2}-\left|u_{n-1}-p\right|^{2}\right]_{+}+2\left|e_{n}\right|^{2}+2\left|e_{n}\right|\left|u_{n}-p\right|$.

Summing up from $n=1$ to $k$, we get

$\sum_{n=1}^{k} \lambda_{n+1}^{2}\left|A u_{n+1}\right|^{2} \leq \sum_{n=1}^{k}\left(\left|u_{n}-p\right|^{2}-\left|u_{n+1}-p\right|^{2}\right)+3 \sum_{n=1}^{k} \alpha_{n}\left|u_{n}-u_{n-1}\right|^{2}+$ $\alpha \sum_{n=1}^{k}\left[\left|u_{n}-p\right|^{2}-\left|u_{n-1}-p\right|^{2}\right]_{+}+2 \sum_{n=1}^{k}\left|e_{n}\right|^{2}+2 \sum_{n=1}^{k}\left|e_{n}\right|\left|u_{n}-p\right|$.

Letting $k \rightarrow \infty$, by part (b) of Proposition 2.1 and the assumptions, we obtain

$$
\sum_{n=1}^{\infty} \lambda_{n+1}^{2}\left|A u_{n+1}\right|^{2}<+\infty
$$

which implies $\liminf _{n}\left|A u_{n}\right|=0$. Since by Lemma 3.1, $\lim _{n}\left|A u_{n}\right|$ exists, thus $\lim _{n}\left|A u_{n}\right|=0$. Now if $u_{n_{j}} \rightarrow q$, then by demicloseness of $A$, we have $q \in A^{-1}(0)$, hence $\omega_{w}\left(u_{n}\right) \subset A^{-1}(0)$. By a similar proof of that of Theorem 2.4 , we can show $\omega_{w}\left(u_{n}\right)$ is singleton. Therefore $u_{n} \rightarrow p \in A^{-1}(0)$.

Remark 3.6. Theorem 3.5 in exact form $\left(e_{n} \equiv 0\right)$ extends Theorem 2.1 of [2]. Because if $e_{n} \equiv 0, A^{-1}(0) \neq \emptyset$ and $\lambda_{n} \geq \lambda>0$, then $\left(\Lambda_{2}\right)$ is satisfied and

$$
\sum_{n=1}^{\infty} \frac{\alpha_{n}^{2}\left|u_{n}-u_{n-1}\right|^{2}}{\lambda_{n+1}^{2}} \leq \frac{\alpha}{\lambda^{2}} \sum_{n=1}^{\infty} \alpha_{n}\left|u_{n}-u_{n-1}\right|^{2}<+\infty
$$

by $\left(\alpha_{1}\right)$ and $\left(\alpha_{2}\right)$. Then $\left(\alpha_{5}\right)$ is also satisfied. 


\section{Subdifferential Case}

In this section, we establish the weak convergence of the sequence $\left\{u_{n}\right\}$ generated by (1.5) to an element of $A^{-1}(0)$, when $A=\partial \varphi$.

Lemma 4.1. Suppose that $\left\{u_{n}\right\}$ is the bounded sequence generated by (1.5) and $A=\partial \varphi$. If the conditions

$$
\left(\alpha_{7}\right) \sum_{n=1}^{+\infty} \frac{\alpha_{n}^{2}\left|u_{n}-u_{n-1}\right|^{2}}{\lambda_{n+1}}<+\infty \text { and }\left(E_{5}\right) \sum_{n=1}^{+\infty} \frac{\left|e_{n}\right|^{2}}{\lambda_{n+1}}<+\infty .
$$

are satisfied, then there exists $\lim _{n \rightarrow+\infty}\left|A u_{n}\right|$.

Proof. By subdifferential inequality and (1.5), we get

$\lambda_{n+1}\left(\varphi\left(u_{n+1}\right)-\varphi\left(u_{n}\right)\right) \leq\left(\lambda_{n+1} \partial \varphi\left(u_{n+1}\right), u_{n+1}-u_{n}\right)$

$=\left(u_{n}-u_{n+1}+\alpha_{n}\left(u_{n}-u_{n-1}\right)+e_{n}, u_{n+1}-u_{n}\right)$

$=-\left|u_{n+1}-u_{n}\right|^{2}+\left(\alpha_{n}\left(u_{n}-u_{n-1}\right)+e_{n}, u_{n+1}-u_{n}\right)$

$\leq \frac{1}{2}\left|\alpha_{n}\left(u_{n}-u_{n-1}\right)+e_{n}\right|^{2} \leq \alpha_{n}^{2}\left|u_{n}-u_{n-1}\right|^{2}+\left|e_{n}\right|^{2}$.

Hence

$$
\varphi\left(u_{n+1}\right) \leq \varphi\left(u_{n}\right)+\frac{\alpha_{n}^{2}}{\lambda_{n+1}}\left|u_{n}-u_{n-1}\right|^{2}+\frac{\left|e_{n}\right|^{2}}{\lambda_{n+1}} .
$$

By assumptions $\left(\alpha_{7}\right),\left(E_{5}\right)$ and Lemma 1.4, there exists $\lim _{n \rightarrow+\infty} \varphi\left(u_{n}\right)$.

The following proposition is the subdifferential case of Proposition 3.2.

Proposition 4.2. Let $\left\{u_{n}\right\}$ be a bounded sequence generated by (1.5) and $A=\partial \varphi$. If $\left(\alpha_{7}\right),\left(E_{5}\right)$, and the following conditions hold:

$$
\left\{\begin{array}{l}
\left(\Lambda_{1}\right) \sum_{n=1}^{+\infty} \lambda_{n}=+\infty \\
\left(\alpha_{3}\right) \sum_{n=1}^{+\infty} \alpha_{n}\left|u_{n}-u_{n-1}\right|<+\infty \text { or }\left(\alpha_{4}\right) \frac{\alpha_{n}\left|u_{n}-u_{n-1}\right|}{\lambda_{n+1}} \rightarrow 0 \\
\left(E_{1}\right) \sum_{n=1}^{+\infty}\left|e_{n}\right|<+\infty \text { or }\left(E_{2}\right) \frac{\left|e_{n}\right|}{\lambda_{n+1}} \rightarrow 0
\end{array}\right.
$$

then $\lim \varphi\left(u_{n}\right)=\inf _{x \in H} \varphi,(\partial \varphi)^{-1}(0) \neq \emptyset$ and $\omega_{w}\left(u_{n}\right) \subset(\partial \varphi)^{-1}(0)$.

Proof. By Proposition 2.2, $A^{-1}(0) \neq \emptyset$. Assume that $p \in(\partial \varphi)^{-1}(0)$, we have

$\lambda_{n+1}\left(\varphi\left(u_{n+1}\right)-\varphi(p)\right) \leq\left(\lambda_{n+1} \partial \varphi\left(u_{n+1}\right), u_{n+1}-p\right)$

$=\left(u_{n}-u_{n+1}+\alpha_{n}\left(u_{n}-u_{n-1}\right)+e_{n}, u_{n+1}-p\right)$

$=\left(u_{n}-p, u_{n+1}-p\right)+\left(p-u_{n+1}, u_{n+1}-p\right)+\left(\alpha_{n}\left(u_{n}-u_{n-1}\right), u_{n+1}-p\right)+\left(e_{n}, u_{n+1}-p\right)$.

So,

$$
\lambda_{n+1}\left(\varphi\left(u_{n+1}\right)-\varphi(p)\right) \leq \frac{1}{2}\left|u_{n}-p\right|^{2}-\frac{1}{2}\left|u_{n+1}-p\right|^{2}+
$$




$$
\alpha_{n}\left|u_{n}-u_{n-1}\right|\left|u_{n+1}-p\right|+\left|e_{n}\right|\left|u_{n+1}-p\right| .
$$

Summing up both sides of (4.3) from $n=1$ to $n=k$ and dividing by $\sum_{n=1}^{k} \lambda_{n+1}$. Letting $k \rightarrow \infty$, since by Lemma $4.1, \lim _{n \rightarrow+\infty} \varphi\left(u_{n}\right)-\varphi(p)=l$ exists, then

$$
\lim _{k \rightarrow+\infty}\left(\sum_{n=1}^{k} \lambda_{n+1}\right)^{-1} \sum_{n=1}^{k} \lambda_{n+1}\left(\varphi\left(u_{n+1}\right)-\varphi(p)\right)=\lim _{k \rightarrow+\infty}\left(\varphi\left(u_{n}\right)-\varphi(p)\right)=l .
$$

Now the assumptions and Lemma 1.2 implies that $l=0$. Thus $\lim _{k} \varphi\left(u_{k}\right)=\varphi(p)$. Therefore, if $u_{n_{j}} \rightarrow q$, then $\varphi(q) \leq \liminf _{j} \varphi\left(u_{n_{j}}\right)=\varphi(p)$, which implies $q \in(\partial \varphi)^{-1}(0)$. Hence $\omega_{w}\left(u_{n}\right) \subset(\partial \varphi)^{-1}(0)$.

The following theorem shows that in the special case $A=\partial \varphi$, which is important from optimization point of view, the conditions $\left(\Lambda_{2}\right)$ and $\left(\alpha_{5}\right)$ in Proposition 3.2 can be replaced by the weaker conditions $\left(\Lambda_{1}\right)$ and $\left(\alpha_{4}\right)$.

Theorem 4.3. Let $\left\{u_{n}\right\}$ be a bounded sequence generated by (1.5) and $A=$ $\partial \varphi$. Assume that the conditions $\left(\Lambda_{1}\right),\left(\alpha_{1}\right),\left(\alpha_{2}\right),\left(\alpha_{7}\right),\left(E_{1}\right)$ and

$\left(E_{5}\right) \sum_{n=1}^{+\infty} \frac{\left|e_{n}\right|^{2}}{\lambda_{n+1}}<+\infty$ are satisfied. Then $u_{n} \rightarrow p \in(\partial \varphi)^{-1}(0)$ as $n \rightarrow \infty$.

Proof. By Proposition 2.2, $A^{-1}(0) \neq \emptyset$. Assume $p \in(\partial \varphi)^{-1}(0)$, we have $\lambda_{n+1}\left(\varphi\left(u_{n+1}\right)-\varphi(p)\right) \leq\left(\lambda_{n+1} \partial \varphi\left(u_{n+1}\right), u_{n+1}-p\right)$

$=\left(u_{n}-u_{n+1}+\alpha_{n}\left(u_{n}-u_{n-1}\right)+e_{n}, u_{n+1}-p\right)$

$=\left(p-u_{n+1}, u_{n+1}-p\right)+\left(u_{n}-p+\alpha_{n}\left(u_{n}-u_{n-1}\right)+e_{n}, u_{n+1}-p\right)$

$\leq-\frac{1}{2}\left|u_{n+1}-p\right|^{2}+\frac{1}{2}\left|u_{n}-p+\alpha_{n}\left(u_{n}-u_{n-1}\right)+e_{n}\right|^{2}$

$=-\frac{1}{2}\left|u_{n+1}-p\right|^{2}+\frac{1}{2}\left|u_{n}-p\right|^{2}+\frac{\alpha_{n}^{2}}{2}\left|u_{n}-u_{n-1}\right|^{2}+\frac{1}{2}\left|e_{n}\right|^{2}$

$+\left(u_{n}-p, e_{n}\right)+\alpha_{n}\left(u_{n}-u_{n-1}, u_{n}-p\right)+\left(\alpha_{n}\left(u_{n}-u_{n-1}\right), e_{n}\right)$

$\leq \frac{1}{2}\left|u_{n}-p\right|^{2}-\frac{1}{2}\left|u_{n+1}-p\right|^{2}+3 \frac{\alpha_{n}}{2}\left|u_{n}-u_{n-1}\right|^{2}+\frac{\alpha_{n}}{2}\left|u_{n}-p\right|^{2}-\frac{\alpha_{n}}{2}\left|u_{n-1}-p\right|^{2}+$ $\left|e_{n}\right|^{2}+\left|e_{n}\right|\left|u_{n}-p\right|$.

So

$$
\left\{\begin{array}{l}
\lambda_{n+1}\left(\varphi\left(u_{n+1}\right)-\varphi(p)\right) \leq \frac{1}{2}\left|u_{n}-p\right|^{2}-\frac{1}{2}\left|u_{n+1}-p\right|^{2}+3 \frac{\alpha_{n}}{2}\left|u_{n}-u_{n-1}\right|^{2} \\
+\frac{\alpha}{2}\left[\left|u_{n}-p\right|^{2}-\left|u_{n-1}-p\right|^{2}\right]_{+}+\left|e_{n}\right|^{2}+\left|e_{n}\right|\left|u_{n}-p\right|
\end{array}\right.
$$

On the other hand, by Lemma 4.1 , there exists $\lim _{n \rightarrow+\infty}\left(\phi\left(u_{n}\right)-\phi(p)\right)$. Let $\lim _{n \rightarrow+\infty}\left(\phi\left(u_{n}\right)-\phi(p)\right)=l$. Multiplying both sides of (4.4) by $\lambda_{n+1}$, summing up from $n=1$ to $n=k$ and then dividing by $\sum_{n=1}^{k} \lambda_{n+1}$. Letting $k \rightarrow+\infty$ then

$$
\lim _{k \rightarrow+\infty}\left(\sum_{n=1}^{k} \lambda_{n+1}\right)^{-1} \sum_{n=1}^{k} \lambda_{n+1}\left(\phi\left(u_{n}\right)-\phi(p)\right)=l .
$$


Thus by assumptions on $\left\{\lambda_{n}\right\},\left\{\alpha_{n}\right\}$ and $\left\{e_{n}\right\}$ and Lemma 1.2, we get: $l=0$. Therefore $\lim _{n \rightarrow+\infty} \phi\left(u_{n}\right)=\phi(p)$. Now if $u_{n_{j}} \rightarrow q$, then $\varphi(q) \leq \liminf _{j} \varphi\left(u_{n_{j}}\right)=\varphi(p)$ implies $q \in(\partial \varphi)^{-1}(0)$. Hence $\omega_{w}\left(u_{n}\right) \subset$ $(\partial \varphi)^{-1}(0)$. The rest of the proof is similar to that of Theorem 2.4.

Remark 4.4. Obviously $\left(\Lambda_{2}\right) \Rightarrow\left(\Lambda_{1}\right)$, on the other hand by $\left(\alpha_{1}\right),\left(\alpha_{2}\right)$ and $\left(\alpha_{5}\right)$, we have:

$$
\begin{gathered}
\sum_{n=1}^{+\infty} \frac{\alpha_{n}^{2}\left|u_{n}-u_{n-1}\right|^{2}}{\lambda_{n+1}} \leq\left(\sum_{n=1}^{+\infty} \frac{\alpha_{n}^{2}\left|u_{n}-u_{n-1}\right|^{2}}{\lambda_{n+1}^{2}}\right)^{\frac{1}{2}}\left(\sum_{n=1}^{+\infty} \alpha_{n}^{2}\left|u_{n}-u_{n-1}\right|^{2}\right)^{\frac{1}{2}} \\
\leq \alpha^{\frac{1}{2}}\left(\sum_{n=1}^{+\infty} \frac{\alpha_{n}^{2}\left|u_{n}-u_{n-1}\right|^{2}}{\lambda_{n+1}^{2}}\right)^{\frac{1}{2}}\left(\sum_{n=1}^{+\infty} \alpha_{n}\left|u_{n}-u_{n-1}\right|^{2}\right)^{\frac{1}{2}}<+\infty .
\end{gathered}
$$

Then $\left(\alpha_{7}\right)$ is satisfied. This shows that, in subdifferential case and when $e_{n} \equiv 0$, the weak convergence of $u_{n}$ is proved under the weaker assumptions on the parameters.

\section{Strong Convergence}

In this final section, the strong convergence of the bounded sequence $\left\{u_{n}\right\}$ generated by (1.5) to a zero of $A$ is studied under additional assumptions on the maximal monotone operator $A$.

Theorem 5.1. Assume that $\left\{u_{n}\right\}$ is a bounded sequence generated by (1.5). If $(I+A)^{-1}$ is a compact operator and the conditions $\left(\Lambda_{2}\right),\left(\alpha_{1}\right),\left(\alpha_{2}\right)$ and $\left(E_{1}\right)$ are satisfied, then $u_{n} \rightarrow p \in A^{-1}(0)$.

Proof. By (3.4) and the assumptions, we get: $\liminf _{n}\left|A u_{n}\right|=0$. Therefore there exists a subsequence $\left\{A u_{n_{j}}\right\}$ of $\left\{A u_{n}\right\}$ such that $\left|A u_{n_{j}}\right| \rightarrow 0$ and $\left\{u_{n_{j}}+A u_{n_{j}}\right\}$ is bounded. Since $(I+A)^{-1}$ is compact, $\left\{u_{n_{j}}\right\}$ has a strongly convergent subsequence (we denote again by $\left\{u_{n_{j}}\right\}$ ) to $p \in H$. The maximality of $A$ implies that $p \in A^{-1}(0)$. On the other hand, by part (b) of Proposition $2.1, \lim _{n}\left|u_{n}-p\right|$ exists. Hence $u_{n} \rightarrow p \in A^{-1}(0)$.

Lemma 5.2. [11] Assume $\left\{y_{n}\right\}$ is a positive real sequence satisfying the following inequality:

$$
b_{n} y_{n} \leq y_{n-1}-y_{n}+a_{n},
$$

where $\left\{b_{n}\right\}$ and $\left\{a_{n}\right\}$ are positive sequences, then we have:

(i) If $\left\{\frac{a_{n}}{b_{n}}\right\}$ is bounded, then the sequence $\left\{y_{n}\right\}$ is bounded.

(ii) If $\lim _{n} \frac{a_{n}}{b_{n}}=0$, then there exists $\lim _{n} y_{n}$.

(iii) If $\lim _{n} \frac{a_{n}}{b_{n}}=0$ and $\sum_{n=1}^{+\infty} b_{n}=+\infty$, then $\lim _{n} y_{n}=0$. 
Theorem 5.3. Let $\left\{u_{n}\right\}$ be a bounded sequence generated by (1.5) and $A$ be a maximal monotone and strongly monotone operator. If $\left(\Lambda_{1}\right),\left(\alpha_{4}\right)$ and $\left(E_{2}\right)$ are satisfied, then $u_{n} \rightarrow p$, where $p$ is the unique element of $A^{-1}(0)$.

Proof. By Proposition 2.2, $A^{-1}(0) \neq \emptyset$. Assume that $p$ is the single element of $A^{-1}(0)$. By the strong monotonicity of $A$ and (1.5), we get

$$
\alpha \lambda_{n+1}\left|u_{n+1}-p\right|^{2} \leq\left(u_{n}-u_{n+1}+\alpha_{n}\left(u_{n}-u_{n-1}\right)+e_{n}, u_{n+1}-p\right) .
$$

It follows that

$2 \alpha \lambda_{n+1}\left|u_{n+1}-p\right|^{2} \leq\left|u_{n}-p\right|^{2}-\left|u_{n+1}-p\right|^{2}+2 \alpha_{n}\left|u_{n}-u_{n-1}\right|\left|u_{n+1}-p\right|+2\left|e_{n}\right|\left|u_{n+1}-p\right|$.

The theorem follows by the assumptions and Lemma 5.2.

\section{References}

[1] F. Alvarez, H. Attouch, Convergence and asymptotic stabilization for some damped hyperbolic equations with non-isolated equilibria, Control Optim. Calc. Var. 6, (2001) 539-552.

[2] F. Alvarez, H. Attouch, An inertial proximal method for maximal monotone operators via discretization of a nonlinear oscillator with damping, Set-valued Anal. 9, (2001) 3-11.

[3] F. Alvarez, On the minimizing property of a second order dissipative system in Hilbert spaces, SIAM J. Control Optim. 38, (2000) 1102-1119.

[4] H. Attouch, F. Alvarez, The heavy ball with friction dynamical system for convex constrained minimization problems, Optimization ( $\mathrm{Na}$ mur, 1998), 25-35, Lecture Notes in Econom. and Math. Systems,481, Springer, Berlin, 2000.

[5] H. Attouch, X. Goudou, P. Redont, The heavy ball with friction method, I. The continuous dynamical system: Global Exploration of the local minima of a real-valued function by asymptotic analysis of a dissipative dynamical system, Commun. Contemp. Math. 2, (2000) 1-34.

[6] H. Brézis, P.L. Lions, Produits infinis de résolvantes, Israel J. Math. 29, (1978) 329-345.

[7] B. Djafari Rouhani, H. Khatibzadeh, On the proximal point algorithm, J. Optim. Theory Appl. 137, (2008) 411-41\%.

[8] M. Edelstein, The construction of an asymptotic center with a fixed -point property, Bull. Amer. Math. Soc. 78, (1972) 206-208. 
[9] F. Jules, P.E. Maingé, Numerical approach to a stationary solution of a second order dissipative dynamical system, Optimization, 51, (2002) 235-255.

[10] H. Khatibzadeh, Asymptotic behavior of a discrete nonlinear oscillator with damping dynamical system, Adv. Difference Equ. 2011, Art. ID 867136, 9 pp.

[11] H. Khatibzadeh, Some remarks on the proximal point algorithm, J. Optim. Theory Appl. 153, (2012) 769-778.

[12] P.L. Lions, Une méthode itérative de résolution d'une inéquation variationnelle, Israel J. Math. 31, (1978) 204-208.

[13] P.E. Maingé, Convergence theorems for inertial KM-type algorithms, J. Comput. Appl. Math. 219, (2008) 223-236.

[14] B. Martinet, Régularisation d'Inéquations Variationnelles par Ap- proximations Successives, Revue Franćaise d'Informatique et de Recherche Opérationnelle 3, (1970) 154-158.

[15] G. Morosanu, Nonlinear Evolution Equations and Applications, Editura Academiei Romane (and D. Reidel publishing Company), Bucharest, 1988.

[16] R.T. Rockafellar, Monotone operators and the proximal point algorithm, SIAM J. Control Optimization 14, (1976) 877-898.

Hadi Khatibzadeh,

Department of Mathematics,

University of Zanjan,

P.O. Box 45195-313, Zanjan, Iran.

Email: hkhatibzadeh@znu.ac.ir

Sajad Ranjbar,

Zarghan Branch, Islamic Azad University,

Zarghan, Iran.

Email: sranjbar@znu.ac.ir

Corresponding author. 